and rivaroxaban in the remaining studies. In comparisons between rivaroxaban and dabigatran for stroke incidence 16 of $19(84 \%)$ studies showing no difference. Edoxaban was included in only 4 studies overall, without evidence suggestive of differential safety or efficacy compared to other NOACs.

Discussion The absence of large-scale randomized controlled trials directly comparing individual approved NOAC agents is a notable scientific gap. Studies with non-random treatment allocation provide weak evidence that apixaban is more safe and more effective in stroke prevention when compared to dabigatran and rivaroxaban, while few studies investigated comparison of edoxaban with other NOACs. Future studies should seek to directly compare NOAC agents with each other. For new medications undergoing evaluation for approval, regulatory agencies should consider mandating comparative efficacy and safety testing between medications when one or more medications are already approved for a specific indication.

\section{DIABETES MELLITUS AND HEART FAILURE: A COMPLETED QUALITY IMPROVEMENT AUDIT}

\footnotetext{
1,2,3P Creechan, ${ }^{2,3,4}$ A Moore, ${ }^{2,3,4} \mathrm{~A}$ Brennan, ${ }^{2,3,4} \mathrm{~B}$ Wong, ${ }^{2,3,4} \mathrm{~A}$ O'Brien, ${ }^{1,2,3,4} \mathrm{C}$ Halley, 1,2,3,4 M Barrett, ${ }^{1,2,3,4} \mathrm{M}$ Ledwidge, ${ }^{1,2,3,4} \mathrm{~K}$ McDonald. 'University College Dublin School of Medicine, Belfield, Dublin, Ireland; 'St. Vincent's University Hospital, Elm Park, Dublin, Ireland; ${ }^{3}$ St. Michael's Hospital, Dun Laoghaire, Dublin, Ireland; ${ }^{4}$ The HeartBeat Trust, Dublin, Ireland
}

10.1136/heartjnl-2021-ICS.56

Introduction Diabetes mellitus (DM) and heart failure (HF) have substantial overlap in their risk factors, pathophysiology, and increasingly in their management. The DAPA-HF trial showed decreasing rates of cardiovascular death and HF hospitalisation with the use of the sodium-glucose co-transporter 2 inhibitors in patients with HF with reduced ejection fraction (HFrEF). This quality-improvement audit had the dual aims of improving the collection and recording of glycaemic control (HbA1c) while also improving the identification and treatment of diabetes in a HF population.

Methods In the initial audit, the electronic medical records of 200 consecutive patients of the Heart Failure Unit (HFU) were analysed. Patient demographics and HF classification were recorded alongside the date/result of their most recent HbA1c as logged on the St Michael's Hospital blood database. The subsequent accuracy and databasing of this data on the bespoke HFU patient system that referred to during every patient interaction was then interrogated with variations in integrity recorded. Our intervention took place at a weekly multidisciplinary meeting where results of the above were presented together with education on the increasing importance of identifying diabetes in HF management. Emphasis was also placed on the need for timely recording of a patient's recent HbA1c on the HF unit database. One month following the above, the records of a further 200 consecutive patients were collected and analysed using the same methodology as the primary audit.

Results A combined total of 400 patients were included in the two audits with an average age of 72 . Males accounted for $57 \%$ of the cohort. HFrEF accounted for $75 \%$ of the population. $51 \%$ of the population were non-diabetics, $27 \%$ were pre-diabetic, $21 \%$ type 2 diabetics and $1 \%$ were type 1 diabetics. Our primary audit of 200 consecutive patients revealed $35 \%(n=70)$ of patients had a HbA1c result available in the last 3 months. $50 \%(n=35)$ of these had a HbA1c $\geq 48$. Only $3 \%(n=5)$ had the above HbA1c result recorded on the HFU patient record system. Of the 200 patients in the post-intervention audit, $67 \%(n=133)$ had a $\mathrm{HbA1c}$ result in the preceding 3 months i.e. representing a 32\% absolute increase in HbA1c testing. 37\% $(n=73)$ had this result subsequently record on the HFU patient record system i.e. representing a 34\% absolute increase in reporting. Importantly, 11 patients identified in the secondary audit were newly diagnosed as diabetics suggesting that these new diagnoses were largely secondary to the increased HbA1c testing that occurred as a result of our intervention.

Summary/Conclusions We present a completed quality-improvement audit cycle showing a significant increase in HbA1c testing and recording in a busy $\mathrm{HF}$ clinic. The data obtained from accurate and consistent $\mathrm{HbA} 1 \mathrm{c}$ recording can be used to identify, treat and accurately monitor diabetic control in the HF population.

\section{IS INTRACARDIAC ECHO GUIDED LEFT ATRIAL APPENDAGE OCCLUSION A SAFE AND COST EFFECTIVE ALTERNATIVE TO TRANSOESOPHAGEAL GUIDED LEFT ATRIAL APPENDAGE OCCLUSION? A SINGLE CENTRE RETROSPECTIVE REVIEW}

K Millar, R Gallen, Y Goh, H Muradagha, M McKittrick, T Kiernan, S Arnous. University Hospital Limerick, Limerick, Ireland

\subsection{6/heartjnl-2021-ICS.57}

Background Left atrial appendage occlusion (LAAO) can be performed in patients with atrial fibrillation with a contraindication to oral anticoagulation to minimise stroke risk. LAAO is usually performed under transoesophageal echocardiography (TOE) guidance. This is labour intensive and requires general anaesthetic (GA). Intracardiac echocardiography(ICE) guided LAAO under conscious sedation may be a safe alternative to TOE guided LAAO. Given the lack of availability of anaesthetic support during the Covid-19 pandemic and the fact that TOE guided LAAO is an aerosol generating procedure, we switched to performing all of our LAAOs under ICE guidance last year. We are the only centre in the country to perform LAAO under ICE guidance. The aim of this retrospective study is to compare the results of ICE guided and TOE guided LAAO procedures.

Methods We retrospectively analysed all of the medical, procedural and outpatient notes and imaging results of patients who underwent LAAO in our centre between January 2018 and January 2021. The co-primary endpoints were successful implantation of the LAA occlusion device and procedure related mortality. The secondary endpoints were; 1) time spent under fluoroscopy; 2) procedure duration; 3) hospital length of stay; 4) incidence of access site haematomas; 5) incidence of pericardial effusion; 6) incidence of tamponade; 7) device embolisation and 8) peri-device leak on follow up TTE or ECG Gated CT Aorta [figure 1].

Results 49 patients were admitted to our centre for LAA occlusion in the study period. They were all included in our study. $12(24.49 \%)$ were female. The median age was 78 with an Interquartile Range (IQR) of 10 . The most common indication for LAA occlusion was gastrointestinal bleeding 
(28 patients, 57.14\%) [table 1]. The co-primary endpoint of successful implantation of the LAA occlusion device occurred in 24 patients (96\%) in the ICE group compared to 24 patients $(100 \%)$ in TOE guided LAAO group $(96 \%$ vs. $100 \%, P=1.00)$. There were no incidences of procedure

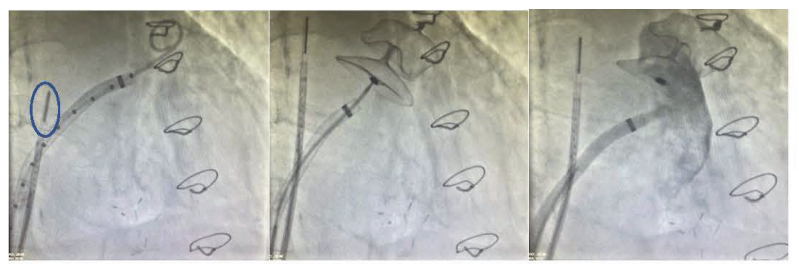

Abstract 57 Figure 1 (Left to Right): IA Fluroscopic Image Showing ICE Catheter (Circled) On Left. IB: ICE Catheter on Left, Amplatzer Delivery Sheath On Right Deploying Amulet LAAO Device. 1C Contrast Image Post Deployment of LAAO Device Showing No Opacification of Left Atrial Appendage with Contrast, Indicative of Satisfactory Occlusion of LAA

Abstract 57 Table 1 Baseline patient characteristics

\begin{tabular}{lll}
\hline & ICE Guided $(\mathbf{n}=25)$ & TOE Guided $(\mathbf{n}=\mathbf{2 4})$ \\
\hline Age & $76 \pm 6.07$ & $78.58 \pm 6.63$ \\
Sex M(\%)/F(\%) & $18(72 \%) / 7(28 \%)$ & $19(79 \%) / 5(21 \%)$ \\
CHA $_{2}$ DS $_{2}$-VASC & $3.84 \pm 1.08$ & $3.75 \pm 0.92$ \\
HASBLED & $2.96 \pm 0.72$ & $2.875 \pm 0.88$ \\
Baseline Haemoglobin & $12.53 \pm 1.69$ & $13.175 \pm 1.48$ \\
Baseline Creatinine & $91.72 \pm 22.21$ & $100.5 \pm 28.57$ \\
Indication for LAA occlusion: & & \\
-Intracranial Haemorrhage (\%) & $9(36 \%)$ & $3(12.5 \%)$ \\
-GI Bleeding (\%) & $13(52 \%)$ & $15(62.5 \%)$ \\
-Epistaxis (\%) & $1(4 \%)$ & $1(4.2 \%)$ \\
-Stroke on NOAC Therapy (\%) & $1(4 \%)$ & $0(0 \%)$ \\
-Haematuria (\%) & $1(4 \%)$ & $2(8.4 \%)$ \\
-Other (\%) & $0(0 \%)$ & $3(12.5 \%)$ \\
\hline
\end{tabular}

\pm Values Refer to Standard Deviation from the Mean Value.

Abstract 57 Table 2 Primary and second endpoints

\begin{tabular}{|c|c|c|c|}
\hline & ICE Guided ( $n=25$ ) & TOE Guided ( $n=24)$ & P Value* \\
\hline \multicolumn{4}{|l|}{ Primary Endpoints: } \\
\hline Successful Implantation (\%) & $24(96 \%)$ & $24(100 \%)$ & 0.312 \\
\hline Procedure Related Death (\%) & $0(0 \%)$ & $0(0 \%)$ & N/A \\
\hline \multicolumn{4}{|l|}{ Secondary Endpoints: } \\
\hline Fluroscopy Time (Mins) & $41.64 \pm 13.39$ & $63.08 \pm 14.93$ & $<0.001$ \\
\hline Procedure Time (Mins) & $127.56 \pm 49.32$ & $184.92 \pm 63.16$ & $<0.001$ \\
\hline Haematoma (\%) & $0(0 \%)$ & $2(8.33 \%)$ & 0.235 \\
\hline LOS & $2.6 \pm 1.55$ & $2.92 \pm 1.15$ & 0.03 \\
\hline Cardiac Arrest (\%) & $0(0 \%)$ & $1(4.16 \%)$ & 1.00 \\
\hline Pericardial Effusion (\%) & $1(4 \%)$ & $1(4.16 \%)$ & 1.00 \\
\hline Tamponade (\%) & $1(4 \%)$ & $0(0 \%)$ & 1.00 \\
\hline Peri-Device Leak (\%) & $1(4 \%)$ & $1(4.16 \%)$ & 1.00 \\
\hline Device Embolisation (\%) & $0(0 \%)$ & $1(4.16 \%)$ & 0.490 \\
\hline
\end{tabular}

related mortality in either group. Mean fluoroscopy time was shorter in the ICE group than the TOE group (Mean $(\mathrm{SD})=41.64 \quad(13.39) \quad$ vs Mean $\quad(\mathrm{SD})=63.08 \quad(14.93) ; 95 \%$ confidence interval (CI)13.30 to 29.59; P<0.001). Mean procedure time was also shorter in the ICE group; (Mean $(\mathrm{SD})=127.56(49.32)$ vs Mean $(\mathrm{SD})=184.92 \quad(63.16) ; 95 \%$ CI 24.86 to $89.85 ; \mathrm{P}<0.001)$. Mean hospital length of stay was lower in the ICE group (2.6 days $(\mathrm{SD}=1.55)$ vs. 2.92 $(\mathrm{SD}=1.15) ; 95 \%$ Confidence Interval (CI) 2.36 to 3.18 ; $\mathrm{P}=0.025)$. There were no statistically significant differences between the groups with regards to the other secondary endpoints [table 2].

Conclusion ICE guided LAA occlusion leads to shorter hospital length of stay, procedure time and reduces time spent under fluoroscopy compared with TOE guided LAA occlusion. There was no statistically significant variations in the procedural complications between the two groups. ICE guided LAA occlusion removes the need for GA, making procedures logistically less challenging and eliminating the need for an aerosol generating procedure (TOE). In the current climate of a global Covid-19 pandemic which has limited access to ICU beds and anaesthetic support, ICE guided LAAO is a safe and cost effective alternative for stroke prevention to TOE guided LAAO in patients with high $\mathrm{CHA}_{2} \mathrm{DS}_{2}$-VASc scores and contraindications to oral anticoagulation. We will continue to follow these patients with gated CT Aorta to ascertain the incidence of device leak.

\section{IMPROVING EFFICACY AND SUSTAINABILITY OF VIRTUAL CARDIOLOGY CLINICS}

CS Gracias, G Fitzgerald, F O'Herlihy, MC Walsh, A Casey, K Hewitt, I Yearoo. Beaumont Hospital, Dublin, Ireland

\subsection{6/heartjnl-2021-ICS.58}

Introduction Social distancing measures were introduced in Ireland to address COVID-19 in March 2020. Strategies to continue Cardiology outpatient services needed to balance patient safety with the need for high quality follow-up care. Therefore, virtual consultations (via phone or video call) were implemented as temporary alternatives to in-person clinics.

Objectives and Methodology We aimed to identify inefficiencies in physical outpatient services at Beaumont Hospital in comparison with virtual consultations. In face-to-face visits, a patient's average time outside the physician's examination room was 39 minutes (range 12-110 minutes). Increased delays occurred if investigations were awaited. The average face-to-face consultation lasted 24 minutes. Next, an analysis was performed on 100 consecutive patients' virtual consultation experiences. Electronic survey responses were collected from stakeholders including patients, physicians, nurses, and clerical staff relating to these virtual visits.

Results $81 \%$ of outpatient consultations were delivered virtually from July to December 2021, with an average duration of 19 minutes. Patient volume was increased $(5$ additional patients per week per clinic) with a reduced waiting time for routine attendance. Criteria for outpatient re-attendance was regularly reviewed, with discharge facilitated at the second or third visit if clinically indicated. $89 \%$ of visits were supported by communications technology (smartphone or internet access) 